\title{
IgA Nephropathy, Infection-associated
}

National Cancer Institute

\section{Source}

National Cancer Institute. IgA Nephropathy, Infection-associated. NCI Thesaurus. Code C123141.

IgA nephropathy co-occurring with infectious disease. 